\title{
Síndrome carcinoide y compromiso valvular cardíaco
}

\author{
Alejandro Paredes, Martín Valdebenito, Víctor Neira, Pablo Castro, Ricardo Zalaquett. \\ Departamento de Enfermedades Cardiovasculares, Pontificia Universidad Católica de Chile.
}

Resumen: El carcinoide es un tumor neuroendocrino raro que se origina típicamente en el tracto gastrointestinal y que puede resultar en un amplio espectro de síntomas mediado por sustancias vasoactivas. La enfermedad carcinoide con compromiso cardíaco es caracterizada por el depósito de tejido fibroso en forma de placas, afectando más frecuentemente las válvulas cardíacas derechas y el endocardio. El compromiso valvular izquierdo ocurre en menos del 10\% de los casos y es casi siempre asociado a un cortocircuito de derecha a izquierda o a un carcinoide bronquial primario. Los pacientes con falla cardíaca derecha sintomáticos tienen limitadas opciones terapéuticas diferentes a la cirugía cardíaca. El reemplazo valvular cardíaco es el único tratamiento efectivo para la enfermedad valvular carcinoide y debiese ser considerada en pacientes sintomáticos, en los cuales la enfermedad metastásica y los síntomas del síndrome carcinoide se encuentran bien controlados. Por estos motivos, se recomienda un enfoque multidisciplinario en un centro de experiencia para este tipo de pacientes.

El presente artículo es una revisión actualizada de la literatura al respecto, tratando de clarificar las principales dudas con respecto al manejo de estos pacientes.

Correspondencia:

Alejandro Paredes C.

Departamento de Enfermedades Cardiovasculares.

Hospital Pontificia Universidad Católica de Chile.

Marcoleta \#367, $7^{\circ}$ piso.

faparedes@puc.cl 


\section{Carcinoid syndrome and heart valve involvement:}

Carcinoid is a rare neuroendocrine tumor typically originating in the gastrointestinal tract and which may result in a broad spectrum of symptoms mediated by vasoactive substances. Carcinoid heart disease is characterized by plaque-like deposits of fibrous tissue, most frequently affecting the right heart valves and endocardium. Left-sided valve disease occurs in less than $10 \%$ of patients with cardiac involvement and is almost always associated with an atrial right-to-left shunt or a primary bronchial carcinoid. Patients with symptomatic right heart failure have limited therapeutic options other than cardiac surgery. Cardiac valve replacement is the only effective treatment for carcinoid heart disease and should be considered for symptomatic patients whose metastatic carcinoid disease and symptoms of carcinoid syndrome are well controlled. A multidisciplinary approach at an experienced center is recommended for the care of these patients

Key Words: carcinoid, carcinoid syndrome, valvular heart diseases.
Introducción: El carcinoide es un tumor neuroendocrino raro que se origina típicamente en el tracto gastrointestinal (intestino delgado distal y colon proximal) y a nivel bronquial, que puede dar origen a un amplio espectro de síntomas mediado por sustancias vasoactivas, que se conoce como síndrome carcinoide ${ }^{1}$. Presenta una incidencia entre 1,2 a 2,1 por 100 mil personas por año ${ }^{2}$.

Las manifestaciones más comunes incluyen cambios vasomotores (flushing $-80 \%$ de los casos), hipermotilidad gastrointestinal (diarrea secretora - 76\% de los casos), broncoespasmo e hipotensión.

La enfermedad carcinoide cardíaca ocurre en alrededor del $50 \%$ y hasta $70 \%^{3}$ de los pacientes con síndrome carcinoide y puede ser la presentación inicial de la enfermedad carcinoide en alrededor del $20 \%$ de los pacientes ${ }^{4}$.

\section{Fisiopatología}

Los mecanismos potenciales de la enfermedad valvular carcinoide incluyen el complejo rol de la serotonina y su interacción con los receptores/transportadores serotoninérgicos. El compromiso ocurriría cuando la serotonina y otras sustancias activas (5-hidroxitriptofano, bradiquininas, taquiquininas, histamina y prostaglandinas) son liberadas a partir de metástasis hepáticas o tumores carcinoides primarios, saltándose el metabolismo hepático. Esto es, debido a que el hígado es capaz de inactivar las sustancias vasoactivas secretadas a la circulación portal; sin embargo, el síndrome carcinoide puede desarrollarse cuando hay metástasis hepáticas que secretan dichas sustancias a la circulación sistémica. Los carcinoides extraintestinales, incluyendo los bronquiales tienen la habilidad de secretar vasoactivos directamente a la circulación sistémica resultando en un síndrome carcinoide en ausencia de enfermedad metastásica ${ }^{5}$.

La enfermedad carcinoide con compromiso cardíaco y valvular se caracteriza por la formación de placas y depósito de tejido fibroso endocárdico primariamente a nivel del corazón derecho ${ }^{6}$ (Figura 1).

La enfermedad cardíaca y valvular por carcinoide no es infrecuente en pacientes con síndrome carcinoide, aumentando de manera considerable la morbimortalidad ya existente. Típicamente compromete la válvula tricúspide con formación de placas carcinoides en ambas superficies de los velos, causando adherencia al endocardio mural y generando un sustrato para la insuficiencia valvular. A nivel pulmonar, el tejido fibroso causa constricción anular, resultando típica- 
Figura 1. Localización de las placas fibrosas en la enfermedad valvular carcinoide.

$\mathrm{VCS}=$ vena cava superior. $\mathrm{VCl}=$ vena cava inferior. VIn= vena innominada. VT= válvula tricúspide. VD= ventrículo derecho. VI= ventrículo izquierdo. Adaptado de Roberts WC. Am J Cardiol 1997; 80:251-56.

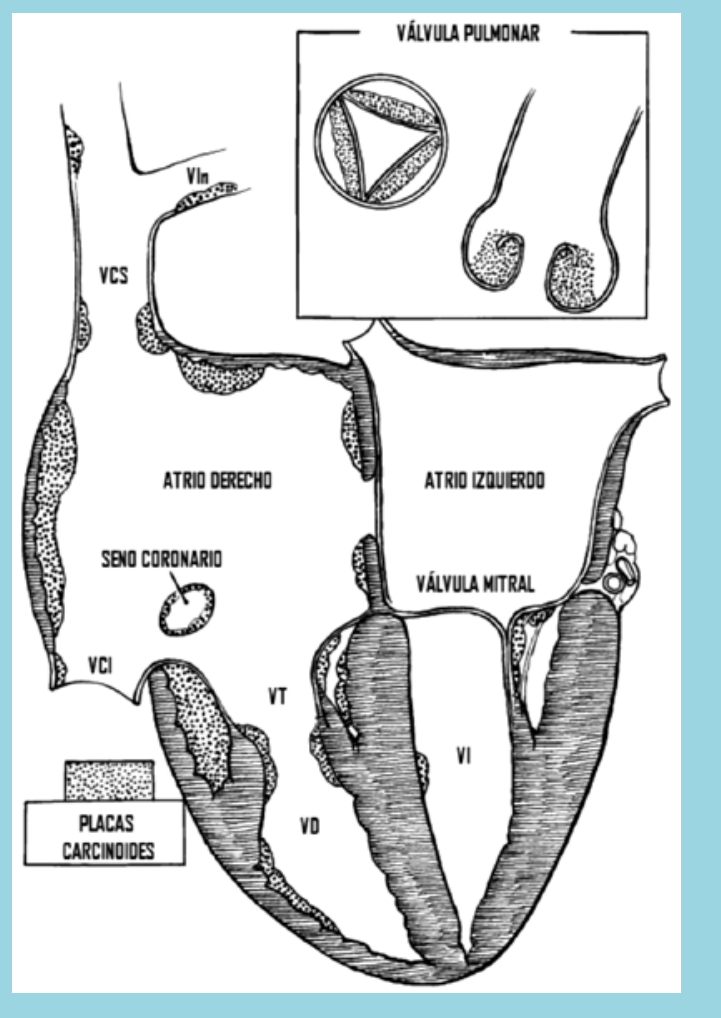

mente en estenosis, aunque también puede determinar insuficiencia. Ambas afecciones valvulares terminan causando falla cardíaca derecha en sus distintos grados.

El compromiso valvular izquierdo es menos frecuente ( $<10 \%$ de los pacientes), pudiendo ocurrir en portadores de comunicación interauricular o foramen oval permeable. Sin embargo, también puede existir compromiso izquierdo en ausencia de cortocircuito por la presencia de carcinoide broncopulmonar o niveles elevados de serotonina circulante. Aunque la morfología de los velos valvulares típicamente no se encuentra alterada, los depósitos de placas y el engrosamiento valvular resultan en disfunción valvular a través de la retracción y fijación de los velos.

\section{Diagnóstico y papel de la ecocardiografía}

El diagnóstico del síndrome carcinoide es usualmente sospechado por los hechos clínicos anteriormente mencionados y confirmado por la identificación del tumor primario, localización de lesiones metastásicas y detección de aumento de productos del metabolismo de la serotonina (sangre u orina) (Figura 2). Dentro de estos, el que presenta mayor sensibilidad y especificidad (cercana al 90\%) es la medición de ácido 5-hidroxiindolacético (5-HIAA) en orina/24 hr .

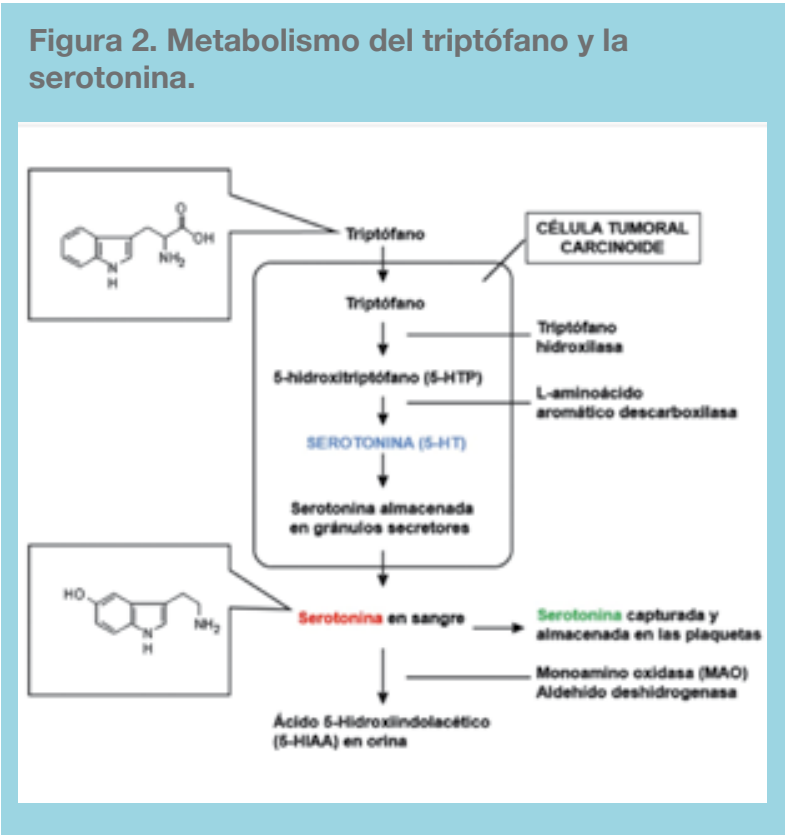

Los niveles elevados de Cromogranina $\mathrm{A}(\mathrm{CgA})$, si bien son sensibles, carecen de mayor especificidad por lo que su utilidad radica en la evaluación de recurrencia y progresión de la enfermedad ${ }^{8}$.

Los péptidos natriuréticos son liberados en respuesta a la expansión de volumen e incremento del estrés parieta ${ }^{19}, \mathrm{y}$ pueden ser útiles en la identificación del compromiso cardíaco en aquellos pacientes con carcinoide, otorgando un peor pronóstico en el seguimiento ${ }^{10}$.

Korse y cols, al estudiar 102 pacientes con tumores neeuroendocrinos y síndrome carcinoide, encontraron que los niveles de CgA y NT-proBNP guardaban relación independiente con la mortalidad general. La sobrevida a 5 años fue de $81 \%$ en los pacientes con niveles normales de $\mathrm{CgA}, 44 \%$ en aquellos con elevación de $\mathrm{CgA}$ pero niveles normales de NT-proBNP, y de $16 \%$ en aquellos con elevación tanto de CgA y NT-proBNP ${ }^{11}$.

La ecocardiografía transtorácica (ETT) se mantiene como la herramienta imagenológica más importante para evaluar la enfermedad valvular carcinoide. Un ETT debe ser realizado en todos los pacientes con diagnóstico de carcinoide. De este modo podremos evaluar las estructuras más comúnmente afectadas incluyendo las válvulas tricúspide y pulmonar ${ }^{12}$, además de estimar el tamaño y función sistólica ventricular 
derecha. La gran mayoría de los pacientes presentará insuficiencia tricuspídea, de los cuales un $90 \%$ será moderada a severa ${ }^{4}$.

De modo similar a la tricúspide, la válvula pulmonar también puede presentarse engrosada y con retracción e inmovilidad de sus velos. Puede encontrarse, además, derrame pericárdico leve en hasta el $10 \%$ de los casos.

\section{Manejo y tratamiento}

Se recomienda un enfrentamiento multidisciplinario en un centro con experiencia en pacientes con enfermedad carcinoide cardíaca y metastásica ${ }^{13}$. Los objetivos terapéuticos son la reducción de los síntomas, mejoría en la calidad de vida, mejoría en la sobrevida por inhibición hormonal o reducción de la carga tumoral.

El agente más efectivo para el alivio de los síntomas en el síndrome carcinoide son los análogos de las somatostatina (ej: Octreotide), los cuales inhiben la secreción de aminas biológicamente activas mediante la unión a receptores de somatostatina en las células carcinoide ${ }^{14}$. Sin embargo, no mejoran la sobrevida de los pacientes ${ }^{15,16}$.

La terapia médica consiste en la reducción de los síntomas de falla cardíaca derecha mediante el uso de diuréticos de asa y/o tiazídicos. El uso de digoxina podría jugar un rol en esta misma línea, pero no hay datos clínicos convincentes que recomienden su indicación generalizada ${ }^{1}$.

Sin tratamiento la mediana de sobrevida con síndrome carcinoide metastásico varía de 12 a 38 meses desde el inicio de los síntomas sistémicos ${ }^{17}$. Muchos pacientes sobreviven por varios años después de la resección del tumor carcinoide primario o se encuentran en tratamiento paliativo por enfermedad metastásica.

La cirugía cardíaca se mantiene como la opción de tratamiento más efectiva para la enfermedad valvular, con marcada mejoría sintomática, $>1$ clase funcional de la NYHA ${ }^{18}$. Cuando existe compromiso cardíaco la sobrevida a 3 años es de $31 \%$, comparada al $68 \%$ sin la afección en cuestión. Se ha descrito un aumento en la sobrevida de 1.5 a 4.4 años en las últimas dos décadas con los avances en el manejo médico-quirúrgico ${ }^{4}$. Dentro de las posibles causas estarían el uso más frecuente de cirugía valvular, citorreducción quirúrgica del tumor primario, terapia ablativa de las metástasis hepáticas y el uso de análogos de somatostatina.

\section{Algunas interrogantes...}

Cabe mencionar algunas interrogantes no resueltas: 1) ¿Cuál es el momento y el riesgo quirúrgico?,2) ¿Qué es más recomendable: plastía valvular, prótesis biológicas o mecánicas?,
3) ¿Qué consideraciones perioperatorias debo tener?.

La primera cirugía valvular por compromiso cardíaco en el carcinoide fue en 1963. La mortalidad perioperatoria fue reducida de $25 \%$ en la década de 1980 a $9 \%$ en los últimos años ${ }^{19}$. El riesgo quirúrgico, aunque en disminución, sigue siendo mayor que el de la valvulopatía reumática o degenerativa. El porcentaje de pacientes intervenidos por compromiso valvular aumentó de 18 a 64\%. Un hecho constante es que la progresión de la insuficiencia cardíaca aumenta aún más la mortalidad perioperatoria ${ }^{20}$.

El reemplazo valvular es la opción de tratamiento más efectiva para la enfermedad valvular carcinoide. Connolly et al., sugieren el reemplazo valvular pulmonar asociado al reemplazo tricuspídeo, lo que ha demostrado reducir el tamaño ventricular derecho después de la cirugía, comparado con el reemplazo valvular tricuspídeo aislado ${ }^{21}$.

Bhattacharyya et al., en su experiencia reportaron como criterios de derivación para cirugía 1) tumor carcinoide estable, 2) disfunción valvular severa, 3) sintomáticos, y 4) ausencia de comorbilidades significativas ${ }^{22}$. Hay quienes señalan que estaría indicada en presencia de valvulopatía que determine falla cardíaca derecha sintomática o dilatación/disfunción sistólica ventricular derecha progresiva asintomática ${ }^{1},{ }^{21}$. Ocasionalmente, la cirugía incluye reemplazo multivalvu$\operatorname{lar}^{23}$ y/o reparación valvular izquierda, cierre de foramen oval permeable, resección de metástasis carcinoides miocárdicas o cirugía de reconstrucción del tracto de salida ventricular derecho.

Inicialmente se recomendó la sustitución de válvulas lesionadas por prótesis mecánicas, en razón del temor de la degeneración prematura de la bioprótesis y el depósito de placas carcinoides sobre ésta ${ }^{24}$. Las prótesis mecánicas no tendrían el riesgo teórico de probable degeneración inducida por la hormona carcinoide, como se describió inicialmente en algunos reportes. Sin embargo, la anticoagulación posterior obligatoria representa un riesgo considerable de sangrado durante procedimientos adyuvantes como citorreducción o embolización tumoral ${ }^{25}$.

Las prótesis biológicas, por otro lado, presentarían una serie de ventajas, dentro de las que destacan la no necesidad de anticoagulación con menor riesgo de sangrado sobre todo en aquellos con disfunción hepática o que deben ser sometidos a procedimientos para tratar el carcinoide. No hay evidencia que demuestra degeneración significativa producto de las hormonas en este tipo de válvulas, como también establecer una diferencia entre la expectativa de vida del paciente en cuestión v/s la duración de la bioprótesis ${ }^{1},{ }^{20,25}$.

Finalmente, las plastías aisladas no serían opción lógica por 
mantener tejido valvular enfermo. Por estos motivos, la elección entre una prótesis mecánica o biológica debe ser individualizada en conjunto con el paciente y el equipo médico tratante, considerando la historia natural de la enfermedad, durabilidad de la válvula, tamaño de la prótesis, reemplazo uni o multivalvular, riesgo de anticoagulación y futuras intervenciones adyuvantes de la enfermedad de base.

\section{Consideraciones perioperatorias generales}

Durante la anestesia - por uso de relajantes musculares liberadores de histamina por ejemplo - se puede precipitar una crisis carcinoide en pacientes con síndrome carcinoide. Estas crisis se caracterizan por flushing, cambios excesivos en la presión arterial, broncoconstricción, arritmias y compromiso neurológico, que pueden ser fatales. Es fundamental, por lo tanto, el control sintomatológico y "profiláctico" prequirúrgico con uso de octreotide (ya sea en bolos o mediante infusión continua) y monitorización anestésica estricta durante el procedimiento $^{26}$.

\section{Pronóstico}

Además de los signos clínicos de insuficiencia cardíaca derecha, la severidad del compromiso estructural y funcional valvular tricuspídeo y la dilatación/disfunción ventricular derecha, han demostrado ser los predictores pronósticos más importantes en esta patología ${ }^{13}$. Afortunadamente, el pronóstico del síndrome carcinoide metastásico y la enfermedad cardíaca carcinoide han mejorado en las últimas décadas de la mano de mejoras en el manejo médico y tratamiento quirúrgico oportuno.

Un análisis retrospectivo de la Clínica Mayo de 200 casos en casi 20 años demostró que las terapias con somatostatina, embolización arterial y quimioterapia no confieren un significativo aumento en la sobrevida. Adicionalmente, evidenció una mejoría en el pronóstico los últimos años, con disminución en la mortalidad perioperatoria y favorables resultados a largo plazo después de la cirugía de reemplazo valvular. Cuando la cirugía cardíaca fue incluida en el análisis multivariado fue asociada a una reducción del riesgo de 0.48 (95\% intervalo de confianza, 0.31-0.73; $\mathrm{P}<001)^{19}$.

\section{Referencias:}

1. ASKEW JW, CONNOLLY HM. Carcinoid valve disease. Curr Treat Options Cardiovasc Med. 2013; 15: 544-55.

2. MODLIN IM, SANDOR A. An analysis of 8305 cases of carcinoid tumors. Cancer, 1997; 79: 813-29.

3. BHATTACHARYYA S, DAVAR J, DREYFUS G, CAPLIN ME. Carcinoid heart disease. Circulation. 2007; 116: 2860-5.

4. PELLIKKA PA, TAJIK AJ, KHANDHERIA BK, SEWARD JB, CALLAHAN JA, PITOT HC, et al. Carcinoid heart disease. Clinical and echocardiographic spectrum in 74 patients. Circulation. 1993; 87:1188-96.
5. BERNHEIM AM, CONNOLLY HM, PELLIKKA PA. Carcinoid heart disease in patients without hepatic metastases. Am J Cardiol. 2007; 99: 292-4.

6. ROBERTS WC. A unique heart disease associated with a unique cancer: carcinoid heart disease. Am J Cardiol. 1997; 80:251-6.

7. PALANISWAMY C, FRISHMAN WH, ARONOW WS. Carcinoid heart disease. Cardiol Rev. 2012; 20: 167-76.

8. MODLIN IM, GUSTAFSSON BI, MOSS SF, PAVEL M, TSOLAKIS AV, KIDD M. Chromogranin A--biological function and clinical utility in neuro endocrine tumor disease. Ann Surg 
Oncol. 2010; 17: 2427-43.

9. PAREDES A VJ, DE LEÓN A, KANACRI A, CASTRO P, BAEZA R. Utility of biomarkers in Heart Failure. Rev Med Chile 2013; 141: 1560-9.

10. BHATTACHARYYA S, TOUMPANAKIS C, CAPLIN ME, DAVAR J. Usefulness of N-terminal pro-brain natriuretic peptide as a biomarker of the presence of carcinoid heart disease. Am J Cardiol. 2008; 102: 938-42.

11. KORSE CM, TAAL BG, DE GROOT CA, BAKKER RH, BONFRER JM. Chromogranin-A and N-terminal pro-brain natriuretic peptide: an excellent pair of biomarkers for diagnostics in patients with neuroendocrine tumor. J Clin Oncol. 2009; 27: 4293-9.

12. GABRIELLI L, CÓRDOVA S, FASCE F, BUROTTO M, ZALAQUET R. Enfermedad cardíaca por carcinoide. Revista Chilena de Cardiología. 2011; 30: 240-2.

13. BERNHEIM AM, CONNOLLY HM, HOBDAY TJ, ABEL MD, PELLIKKA PA. Carcinoid heart disease. Prog Cardiovasc Dis. 2007; 49: 439-51.

14. KUBOTA A, YAMADA Y, KAGIMOTO S, SHIMATSU A, IMAMURA M, TSUDA K, et al. Identification of somatostatin receptor subtypes and an implication for the efficacy of somatostatin analogue SMS 201-995 in treatment of human endocrine tumors. J Clin Invest. 1994; 93: 1321-5.

15. RINKE A, MULLER HH, SCHADE-BRITTINGER C, KLOSE KJ, BARTH P, WIED M, et al. Placebo-controlled, double-blind, prospective, randomized study on the effect of octreotide LAR in the control of tumor growth in patients with metastatic neuroendocrine midgut tumors: a report from the PROMID Study Group. J Clin Oncol. 2009; 27: 4656-63.

16. QUAEDVLIEG PF, VISSER O, LAMERS CB, JANSSEN-HEIJEN ML, TAAL BG. Epidemiology and survival in patients with carcinoid disease in The Netherlands. An epidemiological study with 2391 patients. Ann Oncol. 2001; 12: 1295-300
17. GODWIN JD, 2ND. Carcinoid tumors. An analysis of 2,837 cases. Cancer. 1975; 36: 560-9.

18. CONNOLLY HM, NISHIMURA RA, SMITH HC, PELLIKKA PA, MULLANY CJ, KVOLS LK. Outcome of cardiac surgery for carcinoid heart disease. J Am Coll Cardiol. 1995; 25: 410-6.

19. MOLLER JE, PELLIKKA PA, BERNHEIM AM, SCHAFF HV, RUBIN J, CONNOLLY HM. Prognosis of carcinoid heart disease: analysis of 200 cases over two decades. Circulation. 2005; 112: 3320-7.

20. GUSTAFSSON BI, HAUSO O, DROZDOV I, KIDD M, MODLIN IM. Carcinoid heart disease. Int J Cardiol. 2008; 129: 318-24.

21. CONNOLLY HM, SCHAFF HV, MULLANY CJ, ABEL MD, PELLIKKA PA. Carcinoid heart disease: impact of pulmonary valve replacement in right ventricular function and remodeling. Circulation. 2002; 106: I51-I6.

22. BHATTACHARYYA S, RAJA SG, TOUMPANAKIS C, CAPLIN ME, DREYFUS GD, DAVAR J. Outcomes, risks and complications of cardiac surgery for carcinoid heart disease. Eur J Cardiothorac Surg. 2011; 40: 168-72.

23. ARGHAMI A, CONNOLLY HM, ABEL MD, SCHAFF HV. Quadruple valve replacement in patients with carcinoid heart disease. J Thorac Cardiovasc Surg. 2010; 140: 1432-4.

24. RIDKER PM, CHERTOW GM, KARLSON EW, NEISH AS, SCHOEN FJ. Bioprosthetic tricuspid valve stenosis associated with extensive plaque deposition in carcinoid heart disease. Am Heart J. 1991; 121: 1835-8.

25. TAKAHASHI H, OKITA Y. Cardiac surgery for carcinoid heart disease. Gen Thorac Cardiovasc Surg. 2011; 59: 777-9.

26. WEINGARTEN TN, ABEL MD, CONNOLLY HM, SCHROEDER DR, SCHAFF HV. Intraoperative management of patients with carcinoid heart disease having valvular surgery: a review of one hundred consecutive cases. Anesth Analg. 2007; 105: 1192-9. 